PROCEEDINGS OF THE

AMERICAN MATHEMATICAL SOCIETY

Volume 34, Number 2, August 1972

\title{
PERMUTABLE PRONORMAL SUBGROUPS
}

\author{
TI YEN
}

\begin{abstract}
A bSTRACr. Let $G$ be a finite solvable group. It is shown that a certain class of pronormal subgroups reducing a given Sylow system is a lattice of permutable subgroups.
\end{abstract}

All groups considered here are finite solvable. A subgroup $V$ of a group $G$ is said to be $p$-normally embedded in $G$ if a Sylow $p$-subgroup $P$ of $V$ is also a Sylow subgroup of $P^{G}$-A subgroup $V$ of $G$ is said to be normally embedded in $G$ if it is $p$-normally embedded for every prime $p$. A normally embedded subgroup is necessarily pronormal [1, Theorem 2.3$]$. We prove the following two theorems:

THEOREM 1. A p-subgroup $P$ of $G$ is a Sylow subgroup of $P^{G}$ if it is a Sylow subgroup of $\left\langle P, P^{x}\right\rangle$ for every $x \in G$.

THEOREM 2. Let $\Sigma$ be a Sylow system of the group $G$. Then the set of normally embedded subgroups reducing $\Sigma$ is a lattice of permutable subgroups.

A Sylow system [2] is a complete set of permutable Hall subgroups. A Sylow system $\Sigma$ of $G$ is said to reduce into a subgroup $U$ if $\{U \cap H \mid H \in \Sigma\}$ is a Sylow system of $U$.

Proof of Theorem 1. We proceed by induction on the order $|G|$. Let $A$ be a minimal normal subgroup of $G$. Since $P A \mid A$ satisfies the hypothesis in $G|A, P A| A$ is a Sylow $p$-subgroup of $(P A)^{G}\left|A=P^{G} A\right| A$. Therefore we may assume that $O_{p^{\prime}}(G)=1$ and $\operatorname{core}(P)=1$. Then $P A$ is a Sylow subgroup of $H=P^{G} A$.

Case 1. $H \neq G$. Then $P$ is a Sylow subgroup of $N=P^{H}$. Since $P$ is a pronormal subgroup of $G$ and a subnormal subgroup of $N_{G}(P A), N_{G}(P) \supseteq$ $N_{G}(P A)$. Then $G=H \cdot N_{G}(P A)=H \cdot N_{G}(P)$ and $\operatorname{core}(N)=\bigcap_{x \in G} N^{x}=$ $\bigcap_{x \in N_{G}(P)} N^{x} \supseteq P$. Hence $P$ is a Sylow subgroup of a normal subgroup of $G$.

Case 2. $H=G$. Since $P$ is a Sylow subgroup of $\left\langle P, P^{x}\right\rangle, A \cap\left\langle P, P^{x}\right\rangle=$ $A \cap P \cap P^{x}$. Therefore $A \cap P=A \cap \operatorname{core}(P)=1$. Hence $A$ lies in the center of

Received by the editors September 30, 1971.

AMS 1969 subject classifications. Primary 2040; Secondary 2058.

Key words and phrases. Pronormal subgroups, permutable subgroups, Sylow systems.

(c) American Mathematical Society 1972 
$P A$ and, by a theorem of Gaschütz [4, Hauptsatz 17.4, p. 121], $A$ is complemented by a subgroup $K$. Let $Q=P A \cap K$. Since $A$ lies in the center of $P A, \operatorname{core}(K)=\bigcap_{a \in A} K^{a} \supseteq Q$. Then $A \cdot \operatorname{core}(K) \supseteq(P A)^{G}=G$ so that $K=$ $\operatorname{core}(K)$ and $A$ lies in the center of $G$. Let $B$ be a minimal normal subgroup of $G$ contained in $K$. Similarly, $B$ is a $p$-subgroup lying in the center of $G$. Then core $(P) \supseteq A B \cap P \neq 1$. This proves the theorem.

Proof of Theorem 2. (1) Let $U, V$ be normally embedded permutable subgroups of $G$. Then $U V$ and $U \cap V$ are normally embedded. By a theorem of Wielandt [4, Satz, 4.6, p. 676], there are Sylow $p$-subgroups $P, Q$ of $U$, $V$ respectively such that $P Q$ is a Sylow subgroup of $U V$. Since $|U V| /|P Q|=$ $(|U| /|P|)(|V| /|Q|)(|P \cap Q| /|U \cap V|)$ is a $p^{\prime}$-number, $P \cap Q$ is a Sylow subgroup of $U \cap V$. On the other hand, $P \cap Q=P \cap\left(P Q \cap Q^{G}\right)=P \cap\left(P^{G} \cap Q^{G}\right)$ is a Sylow subgroup of $P^{G} \cap Q^{G}$. Thus $U \cap V$ is $p$-normally embedded.

(2) If a Sylow system $\Sigma$ of $G$ reduces into permutable subgroups $U$ and $V$ then $\Sigma$ reduces into $U \cap V$ and $U V$. That $\Sigma$ reduces into $U \cap V$ is due to Shamash as quoted in [5, Lemma 2, p. 230]. Let $P$ be a Sylow $p$-subgroup of $G$ belonging to $\Sigma$. Then $P \cap U$ and $P \cap U$ are Sylow subgroups of $U$ and $V$ respectively. Since $\Sigma$ reduces to $U \cap V,|U \cap V| /|P \cap U \cap V|$ is a $p^{\prime}$ number. It follows that

$$
\begin{aligned}
|U V| /|(P \cap U)(P \cap V)| & \\
& =(|U| /|P \cap U|)(|V| /|P \cap V|)(|P \cap U \cap V| /|U \cap V|)
\end{aligned}
$$

is a $p^{\prime}$-number. Hence $(P \cap U)(P \cap V)=P \cap U V$ is a Sylow $p$-subgroup of $U V$.

(3) Let $U, V$ be normally embedded subgroups of the group $G$. If $U$ and $V$ reduce a Sylow system $\Sigma$, then $U$ and $V$ are permutable. We use induction on the order $|G|$. Let $A$ be a minimal normal subgroup of $G$. Then $U A \mid A$ and $V A \mid A$ permute. Then $U$ and $V A$ permute. Since $\Sigma$ reduces into $U V A, G=U V A$. Let $p$ be the prime dividing $|A|$. Suppose that $G$ has a normal $p^{\prime}$-subgroup $B$. Then $U$ and $V B$ permute and $G=U V B$. Let $P$ be the Sylow $p$-subgroup of $G$ belong to $\Sigma$. Then $P=(P \cap U)(P \cap V B)=$ $(P \cap U)(P \cap V) \subseteq U V$, so that $G=U V$. Therefore we may assume that $O_{p^{\prime}}(G)=1$. If $p$ does not divide $|U V|$, then $U V$ is the Sylow $p$-complement of $G$ belonging to $\Sigma$. Therefore we may assume that $p$ divides the order $|U|$. Then $O_{p}(G) \cap(P \cap U)^{G}$ is a nonidentity normal subgroup of $G$ contained in $P \cap U$. Replace $A$ by $O_{p}(G) \cap(P \cap U)^{G}$; we get $G=U V$.

\section{REFERENCES}

1. G. A. Chambers, p-normally embedded subgroups of finite soluble groups, J. Algebra 16 (1970), 442-455. MR 42 \#3174.

2. P. Hall, On the Sylow system of a soluble group, Proc. London Math. Soc. (2) 43 (1937), 316-323. 
3. B. Hartley, On Fischer's dualization of formation theory, Proc. London Math. Soc. (3) 19 (1969), 193-207. MR 39 \#5696.

4. B. Huppert, Endliche Gruppen. I, Die Grundlehren der math. Wissenschaften, Band 134, Spinger-Verlag, Berlin and New York, 1967. MR 37 \#302.

5. A. Mann, $\mathfrak{H}$ normalizers of finite solvable groups, J. Algebra 14 (1970), 312-325. MR 40 \#7354.

6. J. S. Rose, Finite soluble groups with pronormal system normalizers, Proc. London Math. Soc. (3) 17 (1967), 447-469. MR 35 \#2967.

Department of Mathematics, Michigan State University, East lansing, MICHIGAN 48823 